\title{
Determining and Controlling the Surface Properties of Cryo-EM Specimen Supports
}

\author{
Katerina Naydenova ${ }^{1,2}$, Christopher J. Russo ${ }^{1}$ \\ 1. MRC Laboratory of Molecular Biology, Cambridge, United Kingdom \\ 2. Trinity College, University of Cambridge, Cambridge, United Kingdom
}

Electron cryomicroscopy (cryo-EM) has become a widely used method for determining high-resolution structure of biological molecules and complexes. Still, one of the major practical limitations of this technique is specimen preparation. Frequently encountered problems include contamination, formation of too thick, too thin or non-uniform amorphous ice, and adsorption of the specimen to surfaces present in the specimen support just before freezing. All of these problems are influenced by the surface properties of the support and so simple methods for modifying these surfaces using low-energy plasmas have been developed $[1,2]$. The primary goal of these plasma treatment instruments and methods is to improve the wettability of the support structure by decreasing its hydrophobicity and to remove contamination from the support before the specimen is applied.

We studied the effect of argon and oxygen plasma treatments on the surface of copper, gold and amorphous carbon-covered cryo-EM specimen supports and how their properties change with time in different storage conditions. To monitor the state of the surface, we measured the contact angle of microdroplets of deionized water with the support, and calculated the surface energies with the YoungDupré equation [3]. The plasma treatments were performed in a commercial plasma chamber (Fischione, Model 1070 NanoClean) fitted with a custom grid holder [2], and a custom instrument was constructed for accurate measurement of the contact angle directly on an EM specimen support (Figure 1a). The contact angle depends both on the surface chemistry induced by the plasma and on the presence of contaminants.

We demonstrate that inert plasma treatment for less than one minute is sufficient to remove hydrocarbonous contaminants from the surface of the specimen support. Reproducibility of a surface treatment is highly sensitive to the purity of the plasma composition: as an example, presence of residual oxygen in the plasma chamber with partial pressure 1 part in $10^{4}$ in the Ar plasma during a typical cleaning routine results in $\sim 1$ oxygen molecule per $\AA^{2}$ per second encountering the surface. Introduction of oxygen to the plasma composition causes formation of oxidation layers on copper and gold surfaces, which cause an increase of the adhesion energy (Figure 1b) [4]; the rate of oxidation can be controlled by varying the oxygen fraction in the plasma. The rate of amorphous carbon etching was measured by AFM. We found that layers of carbon atoms are removed at a rate of $3 \pm 1 \AA / \mathrm{s}$ (in $90 \% \mathrm{Ar}, 10 \% \mathrm{O}_{2}$ plasma at $21 \mathrm{mbar}$ and $38 \mathrm{~W}$ forward power), which also provides an estimate of the rate of removal of contamination from the surfaces of the supports.

The rate of re-contamination and the change in the surface state of the supports depends both on the surface itself and the storage conditions. We demonstrate that storage of specimen supports in conventional conditions (plastic grid boxes or glass petri dishes) is inadequate for preserving the effects of plasma treatment for times longer than $\sim 1$ hour (Figure 1b). We also show that storage in plastic grid boxes is similar to storage in a hydrocarbon vapour enriched atmosphere in terms of contamination rate. A comparison of different storage conditions suggests high-purity inert atmospheres $\left(\mathrm{N}_{2}\right.$ or $\left.\mathrm{Ar}\right)$ are a suitable long-term storage environment for plasma-treated EM grids, where the lifetime of the treated surfaces is 
prolonged more than 10-fold compared to storage in ambient air.

We interpret our observations in terms of a three-component model of the grid surface, comprised of patches of the native metal, an oxidation layer and adsorbed hydrocarbonous contaminants. Based on the results of this study, we posit that controlled application of low-energy plasmas of ultra-high purity gases can be used to tune the surfaces of cryo-EM specimen, and the effects of the plasma on the support can be measured by a simple contact angle instrument designed to image microdroplets on grids. Storage of the specimen support after plasma treatment is as important as the conditions of the plasma itself, and must be considered when attempting to create reproducible conditions for specimen preparation in cryo-EM [5].

\section{References:}

[1] T C Isabell et al, Microsc Microanal 5 (1999), p. $126-135$

[2] L A Passmore, C J Russo, Methods Enzymol 579 (2016), p. 51 - 86

[3] J N Israelachvili in "Intermolecular and Surface Forces", 3rd ed., (Elsevier, 2011)

[4] M L White, J Drobek, J Phys Chem 70 (1966), p. 3432 - 3436

[5] The authors acknowledge S. Scotcher and the MRC-LMB mechanical workshop.

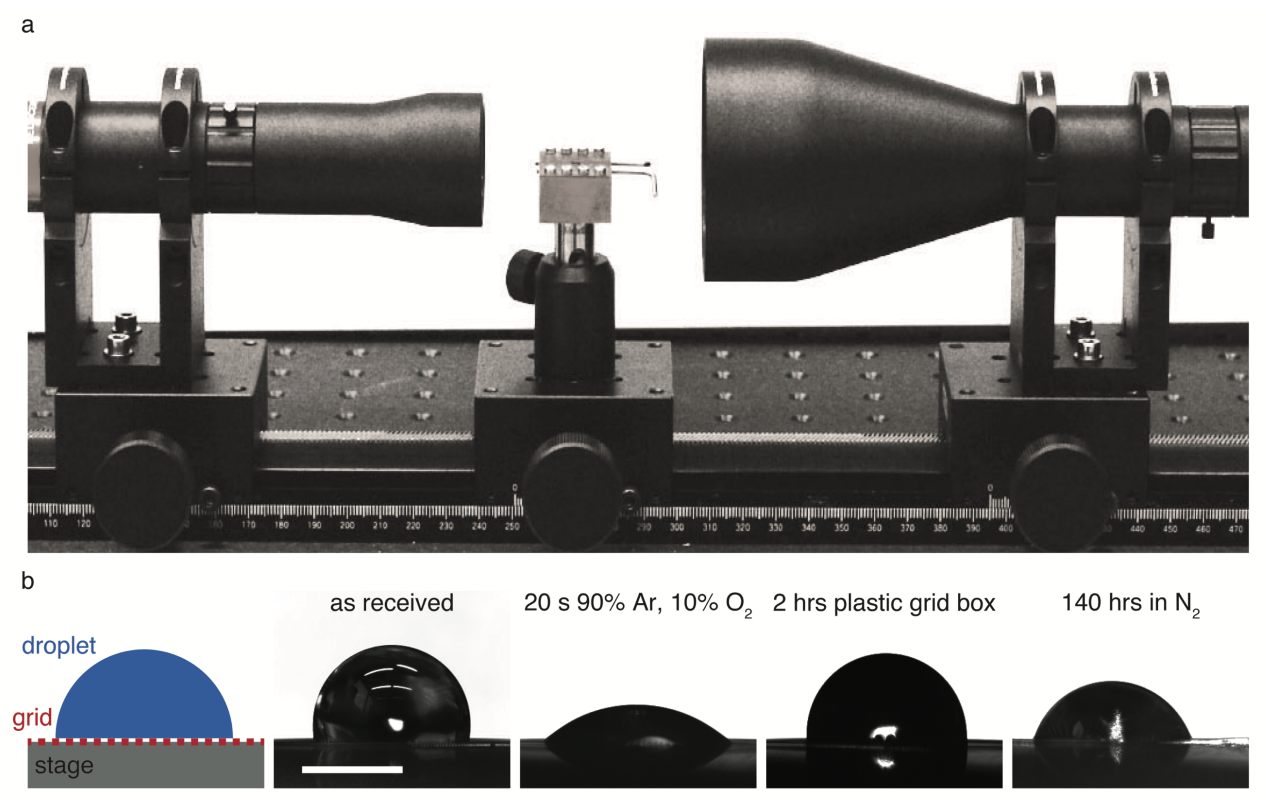

Figure 1. (a) Design of an instrument to measure the contact angle on EM specimen supports. Field of view in image is $36 \mathrm{~cm}$ wide. (b) Contact angle of water on copper grids (from left to right: as received, immediately after plasma treatment, after plasma treatment and storage in a plastic grid box, after plasma treatment and storage under nitrogen). Scale bar is $1 \mathrm{~mm}$ and magnification is the same in each micrograph. 